\title{
WEE1 inhibition induces glutamine addiction in T-cell acute lymphoblastic leukemia
}

Haematologica 2021

Volume 106(7):1816-1827

\section{Correspondence:}

HUDAN LIU

hudanliu@whu.edu.cn

Received: July 2, 2019.

Accepted: January 2, 2020.

Pre-published: January 9, 2020.

https://doi.org/10.3324/haematol.2019.231126

(C)2021 Ferrata Storti Foundation

Material published in Haematologica is covered by copyright. All rights are reserved to the Ferrata Storti Foundation. Use of published material is allowed under the following terms and conditions:

https://creativecommons.org/licenses/by-nc/4.0/legalcode. Copies of published material are allowed for personal or internal use. Sharing published material for non-commercial purposes is subject to the following conditions:

https://creativecommons.org/licenses/by-nc/4.0/legalcode, sect. 3. Reproducing and sharing published material for commercial purposes is not allowed without permission in writing from the publisher.

\section{Juncheng $\mathrm{Hu},{ }^{1,2, *}$ Tianci Wang, ${ }^{1,2, *}$ Jin $\mathrm{Xu},{ }^{2}$ Sanyun $\mathrm{Wu},{ }^{1}$ Liyuan Wang, ${ }^{2}$ Hexiu Su, ${ }^{2}$ Jue Jiang, ${ }^{2}$ Ming Yue ${ }^{3}$ Jingchao Wang, ${ }^{2}$ Donghai Wang, ${ }^{2}$ Peng $\mathrm{Li},{ }^{4}$ Fuling Zhou, ${ }^{1}$ Yu Liu, ${ }^{5}$ Guoliang Qing ${ }^{2}$ and Hudan Liu ${ }^{1,2}$}

${ }^{1}$ Department of Hematology, Zhongnan Hospital, Wuhan University, Wuhan; ${ }^{2}$ Frontier Science Center for Immunology and Metabolism, Medical Research Institute, Wuhan University, Wuhan; ${ }^{3}$ Department of Pharmacy, The Central Hospital of Wuhan, Tongji Medical College, Huazhong University of Science and Technology, Wuhan; ${ }^{4}$ South China Institute for Stem Cell Biology and Regenerative Medicine, Guangzhou Institutes of Biomedicine and Health, Chinese Academy of Sciences, Guangzhou and ${ }^{5}$ Pediatric Translational Medicine Institute, Shanghai Children's Medical Center, Shanghai Jiao Tong University School of Medicine, Shanghai, China

*JH and TW contributed equally as co-first authors.

\section{ABSTRACT}

cell acute lymphoblastic leukemias (T-ALL) are aggressive and heterogeneous hematologic tumors resulting from the malignant transformation of T-cell progenitors. The major challenges in the treatment of T-ALL are dose-limiting toxicities of chemotherapeutics and drug resistance. Despite important progress in deciphering the genomic landscape of T-ALL, translation of these findings into effective targeted therapies remains largely unsuccessful. New targeted agents with significant antileukemic efficacy and less toxicity are urgently needed. Here we report that the expression of WEE1, a nuclear tyrosine kinase involved in cell cycle G2-M checkpoint signaling, is significantly elevated in T-ALL. Mechanistically, oncogenic MYC directly binds to the WEE1 promoter and activates its transcription. T-ALL cells particularly rely on the elevated WEE1 for cell viability. Pharmacological inhibition of WEE1 elicits global metabolic reprogramming which results in a marked suppression of aerobic glycolysis in T-ALL cells, leading to an increased dependency on glutaminolysis for cell survival. As such, dual targeting of WEE1 and glutaminase (GLS1) induces synergistic lethality in multiple TALL cell lines and shows great efficacy in T-ALL patient-derived xenografts. These findings provide mechanistic insights into the regulation of WEE1 kinase in T-ALL and suggest an additional vulnerability during WEE1 inhibitor treatments. We also highlight a promising combination strategy of dual inhibition of cell cycle kinase and metabolic enzymes for T-ALL therapeutics.

\section{Introduction}

T-cell acute lymphoblastic leukemias (T-ALL) are highly proliferative hematologic tumors, ${ }^{1}$ which represent $10-15 \%$ of pediatric and $25 \%$ of adult acute lymphoblastic leukemia cases. ${ }^{2}$ Introduction of intensified chemotherapy protocols in T-ALL treatment significantly improves the overall survival in pediatric patients. ${ }^{3}$ Despite this progress, chemotherapeutic treatments come with significant shortterm and long-term side effects ${ }^{4}$ and the prognosis of patients with resistant or relapsed diseases remains dismal. ${ }^{5}$ Moreover, the remarkable success of pediatric treatment has not been achieved in adult patients as they do not always tolerate intensive pediatric regimens. ${ }^{6}$ Identification of activating mutations in NOTCH1 in over $50 \%$ of T-ALL cases has stimulated much interest in the development of antiNOTCH1 therapies. However, the clinical development of $\gamma$-secretase inhibitors (GSI), which block a critical proteolytic step required for NOTCH1 activation, has been hampered by limited efficacies in human patients and significant gastrointestinal toxicity. Facing these clinical challenges, new tar- 
geted therapies are needed to improve the outcomes of those patients with a poor prognosis and reduce the side effects associated with chemotherapies.

Uncontrolled proliferation is one of the hallmarks of cancer. Many cancer cells possess a deficient G1 cell cycle checkpoint, for example due to p53 loss, and this impairs the ability of cells to halt the cell cycle and repair DNA damage before replication (S-phase). ${ }^{8}$ It provides cancer cells with a means to accumulate mutations and propagate irregularities that are favorable for proliferation. Meanwhile, tumor cells become more reliant on the G2-M cell cycle checkpoint to prevent mitotic entry with excessive DNA damage, which may lead to apoptosis due to mitotic catastrophe. WEE1 is a tyrosine kinase that plays a crucial role as the gatekeeper of the G2-M checkpoint. ${ }^{9}$ When DNA damage occurs, it leads to activation of WEE1 which phosphorylates CDK1 and maintains the CDK1cyclin $\mathrm{B}$ complex in an inactive form, preventing entry into mitosis. ${ }^{10}$ To limit excessive genomic instability in tumor cells, it is not surprising that WEE1 is highly expressed in a variety of cancer types. ${ }^{11-13}$ Moreover, high WEE1 expression has been associated with poor rates of disease-free survival..$^{11,14,15}$ Despite considerable studies on the role of WEE1 in cell cycle checkpoints, it remains unclear how expression of WEE1 is increased and how increased WEE1 expression promotes neoplastic phenotypes.

Cellular metabolism is at the foundation of all biological activities, and altered tumor cell metabolism is now firmly established as another hallmark of human cancer. Normal cells primarily rely on aerobic respiration/oxidative phosphorylation to meet their energy requirements, yet fastgrowing, poorly differentiated tumor cells typically exhibit increased aerobic glycolysis by converting a majority of glucose-derived pyruvate to lactate. ${ }^{16}$ Because of this, tumor cells depend on glutamine anaplerosis to replenish tricarboxylic acid (TCA) cycle intermediates (e.g., $\alpha$-ketoglutarate) to sustain the metabolic integrity and produce nicotinammide adenina dinucleotide (NADH). ${ }^{17}$ Reprogramming of glucose and glutamine metabolism not only provides tumor cells with building blocks for macromolecule biosynthesis but also rescues them from a stressed cellular microenvironment by maintaining proper redox homeostasis. ${ }^{16}$ In T-ALL, both glycolysis and glutaminolysis play crucial roles in mediating leukemia cell proliferation, survival and drug resistance. ${ }^{18,19}$

In this study, we show that the elevated expression of WEE1 kinase in T-ALL results from oncogenic MYC-mediated transcriptional activation, and this WEE1 upregulation significantly contributes to efficient aerobic glycolysis. Pharmacological inhibition of WEE1 leads to a marked decrease in glycolytic flux, rendering T-ALL cells particularly vulnerable to glutamine deficiency. Based on these findings, dual targeting of WEE1 and glutaminase (GLS1), the key rate-limiting enzyme in the glutaminolysis pathway, ${ }^{20}$ shows great promise in anti-T-ALL targeted therapies.

\section{Methods}

\section{Cell culture and reagents}

T-ALL cells were maintained in RPMI-1640 (Hyclone, Logan, UT, USA) supplemented with $10 \%$ FBS (Hyclone) as described. ${ }^{21,22}$ Human primary specimens were obtained with informed consent from Guangzhou Institutes of Biomedicine and Health, Chinese Academy of Sciences and Zhongnan Hospital, Wuhan University, China. Polymerase chain reaction (PCR) primer sequences and antibodies used in this study are listed in Online Supplementary Tables S1 and S2, respectively.

\section{Metabolomic analysis}

HPB-ALL cells were treated with mock (DMSO) or selective WEE1 inhibitor MK1775 ${ }^{23}$ (200 nM) for 20 hours (h). Ten million cells in each treatment were collected and quenched in liquid nitrogen. Metabolite samples were prepared for analysis using standard solvent extraction methods and then subjected to the gas chromatograph system (Agilent Technologies, Santa Clara, CA, USA) coupled with a Pegasus HT gas chromatography timeof-flight mass spectrometer (GC-TOF-MS; LECO Corporation, St Joseph, MI, USA). ${ }^{24,25}$ Identification of chemical entities was based on comparison to Fiehn metabolomics library. Chroma TOF $4.3 x$ software and the LECO-Fiehn Rtx5 database were used for raw peak identification and integration of the peak area. Both mass spectrum match and retention index match were taken into consideration. ${ }^{26}$ Normalized data were uploaded using the SIMCA software package (V14.1, Sartorius Stedim Data Analytics AB, Umea, Sweden) for principal component analysis (PCA) and orthogonal projections for latent structuresdiscriminant analysis (OPLS-DA). Differential metabolites between experimental groups were determined by variable importance in the projection (VIP) values $(\mathrm{VIP}>1)$ and Student $t$ test. The metabolic pathway enrichment analysis was performed using Kyoto Encyclopedia of Genes and Genomes (KEGG, http://www.genome.jp/kegg/) and MetaboAnalyst 4.0 (http://www. metaboanalyst.ca).

\section{Mouse studies}

HPB-ALL xenografts were carried out as previously described. ${ }^{21,27}$ NOD-Prkdcscid IL2R $\gamma^{\text {null }}$ NPG mice (4-6 weeks old, Beijing Vitalstar Biotechnology Co., Beijing, China) were injected with five million cells infected with lentiviruses expressing the green fluorescent protein (GFP) and luciferase (pWPXLdLuciferase-GFP). Mice were subjected to bioimaging at day 6 post engraftment with IVIS Lumina II (Waltham, MA, USA) to ensure equivalent tumor onset in vivo. These animals were then randomly divided into four groups undergoing treatments in a three days on and three days off mode for four cycles. MK1775 $(20 \mathrm{mg} / \mathrm{kg})$ was administered twice daily by oral gavage and BPTES (25 $\mathrm{mg} / \mathrm{kg}$ ) was intraperitoneally injected once daily. Disease progression and therapy response were evaluated by bioimaging.

For drug synergy studies in the patient-derived xenograft (from primary T-ALL sample \#1, Online Supplementary Table S3), T-ALL cells were injected into irradiated 4-6 week old NPG mice (2 Gray), which were subjected to treatment at day 6 post engraftment. Control, MK1775 (20 mg/kg), CB-839 (200 mg/kg) or both were administrated by oral gavage every other day for two consecutive weeks. Leukemia burden was assessed by flow cytometry analysis of human $\mathrm{CD}_{4} 5^{+}$cells. All animal experiments were performed under animal ethical regulations and the study protocol was approved by the Institutional Animal Care and Use Committee of Wuhan University.

\section{Statistical analysis}

Spearman rank correlation test was used to analyze the WEE1 and MYC expression in primary T-ALL samples (Figure $2 \mathrm{~F}-\mathrm{H}$ ). Log-rank analysis was used to evaluate differences in KaplanMeier survival curves. Student $t$-test or one-way ANOVA was used in other statistical analysis. $P<0.05$ was considered statistically significant. 


\section{Results}

\section{WEE1 is highly expressed in T-cell acute lymphoblastic leukemia}

To determine the expression of WEE1 in T-ALL, we analyzed gene expression profiles from three independent T-ALL patient cohorts. ${ }^{28.30}$ WEE1 expression in primary TALL was significantly elevated as compared to normal bone marrow (BM) cells (Figure 1A). Analysis of the Cancer Cell Line Encyclopedia (CCLE) ${ }^{31}$ demonstrated that WEE1 is the third highest expressed in T-ALL among 1,429 human cancer cell lines derived from 40 tumor origins (Online Supplementary Figure S1), suggesting the particular- ly important role of WEE1 in T-ALL. Quantitative PCR analysis verified these findings in ten T-ALL patient samples and eight T-ALL cell lines that WEE1 mRNAs were significantly increased in T-ALL compared with normal BM (Figure 1B and Online Supplementary Table S3). To further correlate the level of WEE1 transcript with its protein expression, we next compared the WEE1 protein expression between normal and transformed thymocytes. Again, WEE1 was generally more abundant in T-ALL cells than normal thymocytes from healthy donors (Figure 1C). Related to our finding, increased WEE1 expression had previously been found in 58 adult T/B-ALL samples as compared to normal mononuclear cells. ${ }^{13}$ We next assessed

A
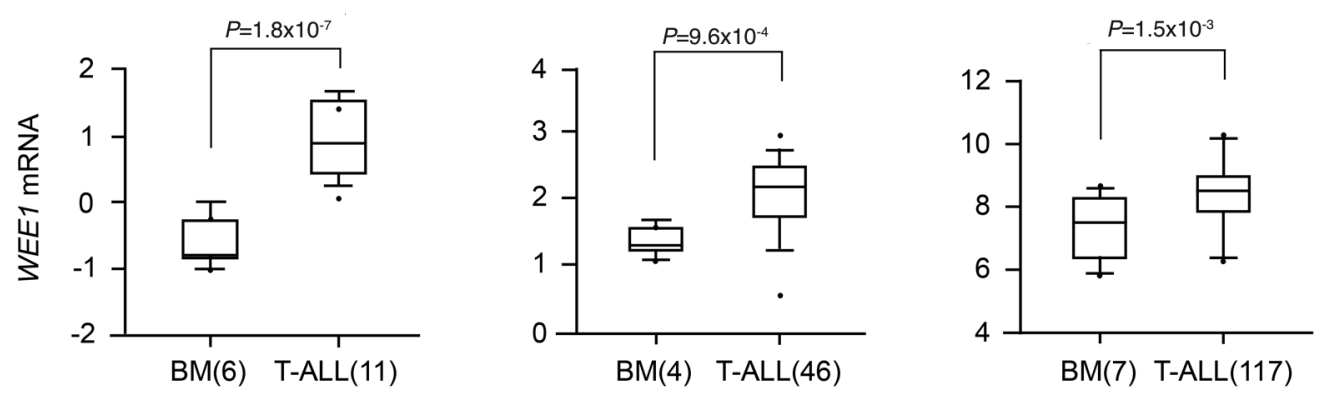

B

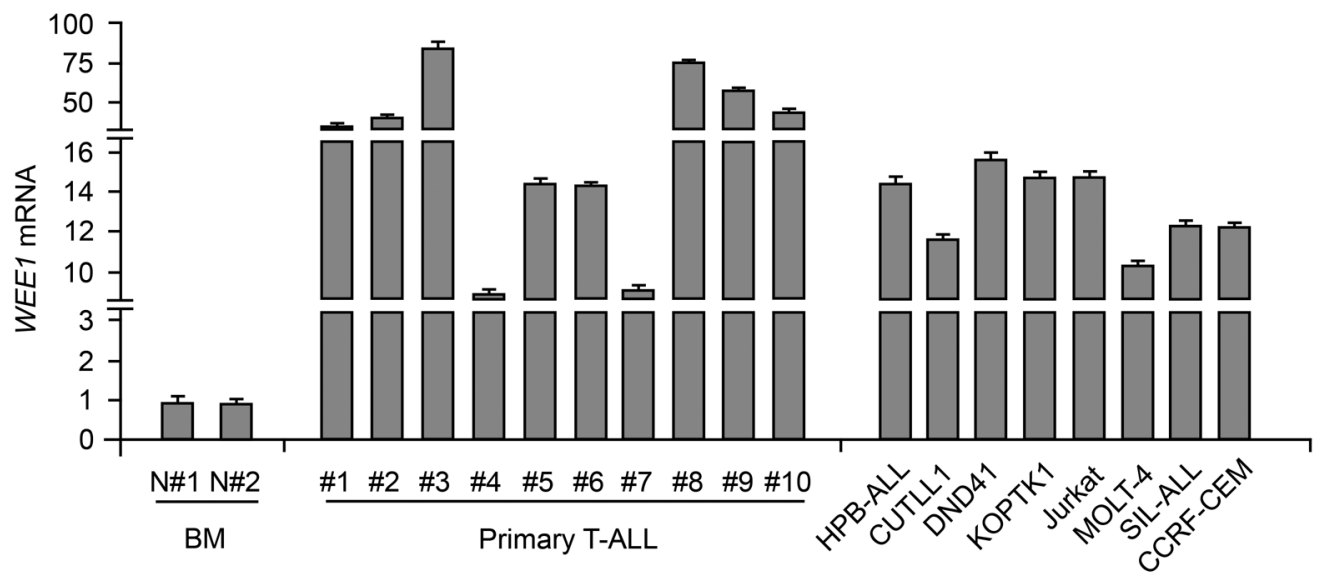

C

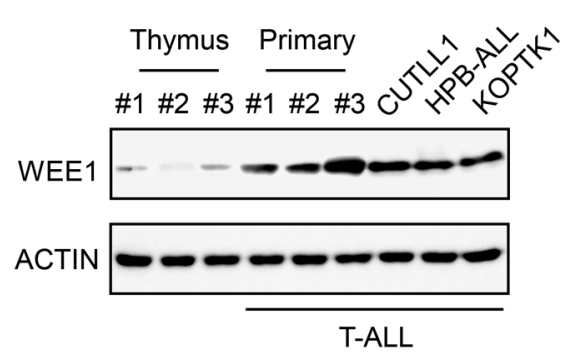

D

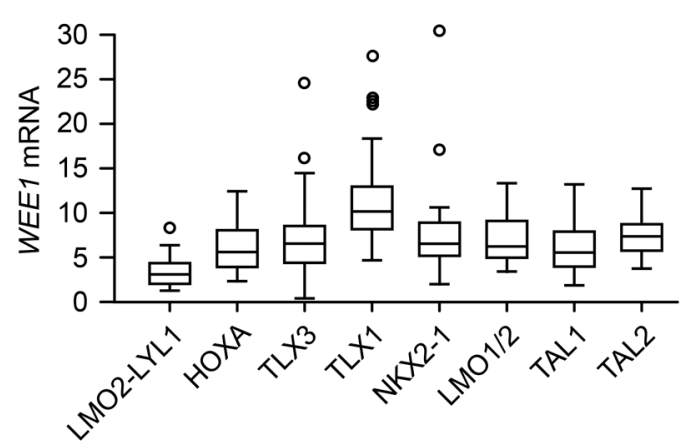

Figure 1. High WEE1 expression in T-cell acute lymphoblastic leukemia (T-ALL). (A) WEE1 mRNA expression was analyzed in primary T-ALL and normal bone marrow (BM) from microarray datasets (left, 6 normal BM and 11 T-ALL samples in GSE7186; middle, 4 normal BM and 46 T-ALL samples in GSE28497; right, 7 normal BM and 117 T-ALL samples in GSE26713). The distributions of WEE1 mRNA expression are presented as Log median-entered intensity and shown in Box-and-Whisker plots with the median value (line), the interquartile range (box), and up to 1.5x the interquartile range (bars). (B) Relative WEE1 mRNA expression in normal BM, primary T-ALL cells and T-ALL cell lines as indicated. (C) Immunoblots of WEE1 in normal human thymus, primary T-ALL cells and T-ALL cell lines as indicated. ACTIN serves as a loading control. (D) Analysis of WEE1 expression in 264 primary T-ALL samples that are categorized into eight different subtypes. $y$-axis denotes WEE1 FPKM values in Log scale from the RNA-sequencing (Seq) dataset. 
WEE1 expression in different T-ALL subtypes from 264 primary T-ALL samples, based on unique gene expression signatures reflecting distinct stages of arrest during T-cell development. ${ }^{32}$ Interestingly, WEE1 mRNA levels varied with the highest expression in the TLX1 subtype (Figure 1D). Regardless of these variations, we identified a global upregulation of WEE1 in T-ALL.

\section{MYC directly activates WEE1 transcription in T-cell acute lymphoblastic leukemia}

To understand the molecular mechanism underlying WEE1 upregulation in T-ALL, we conducted in silico analysis in the UCSC genome browser gateway to identify potential transcription factor regulating WEE1 expression. MYC, TAL1 and GATA3 were predicted to activate the WEE1 promoter (Online Supplementary Figure S2A). We individually knocked down each of these transcription factors, using two individual shRNA (sh\#1 or sh\#2) in human T-ALL cells, and found that only depletion of MYC decreased WEE1 mRNA and protein levels (Figure 2A and Online Supplementary Figure S2B). Consistently, bromodomain 4 (Brd4) inhibitor JQ1, which represses MYC transcription, ${ }^{33}$ decreased WEE1 mRNA and protein levels concomitant with downregulation of MYC expression in multiple T-ALL cells (Figure 2B and Online Supplementary Figure S2C). In contrast, JQ1 treatment, inhibiting N-MYC as well ${ }^{34}$ caused minimal effect on the WEE1 steady-state level in T-ALL LOUCY cells which predominantly express N-MYC (Online Supplementary Figure S2D). In addition, inactivation of MYC similarly down-regulated WEE1 (expression in Burkitts lymphoma P493 cells (Online Supplementary Figure S2E). These data suggest that MYC,
A

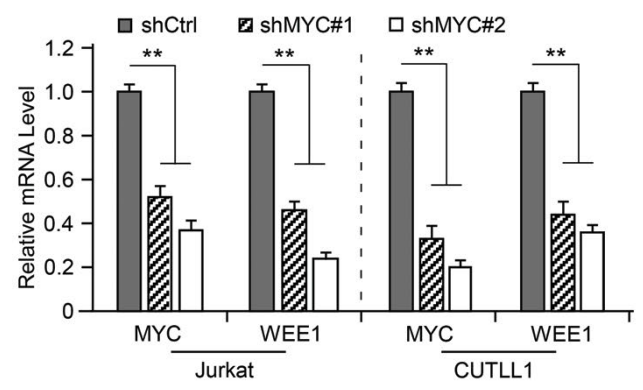

B

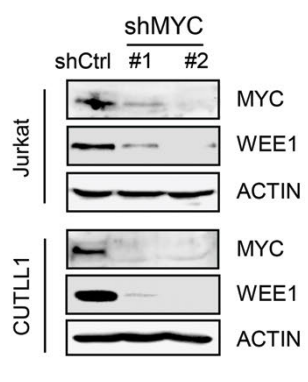

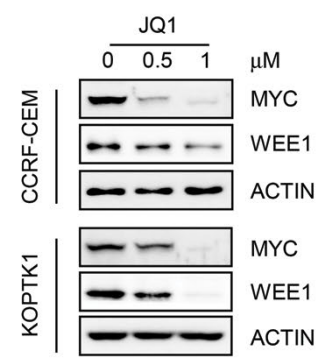

C

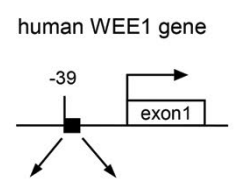

cactCACGTGgtta WT

cactAACTTCgtta MUT
D

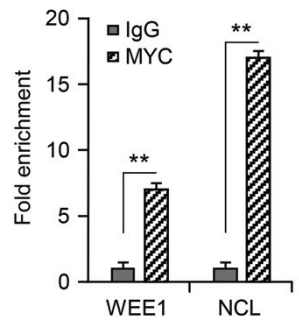

E

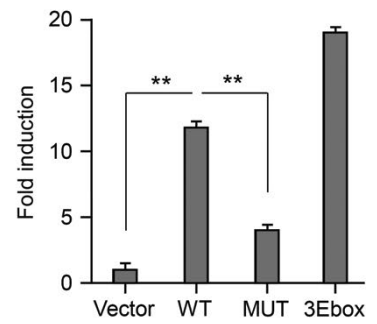

$\mathrm{F}$

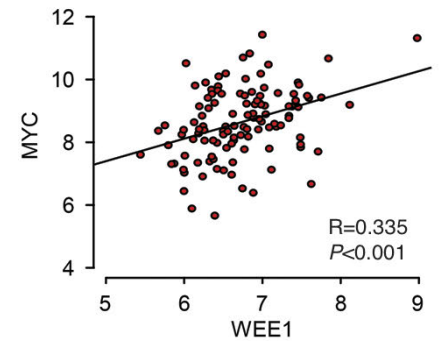

G

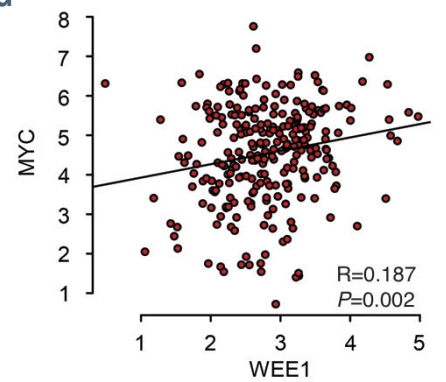

H

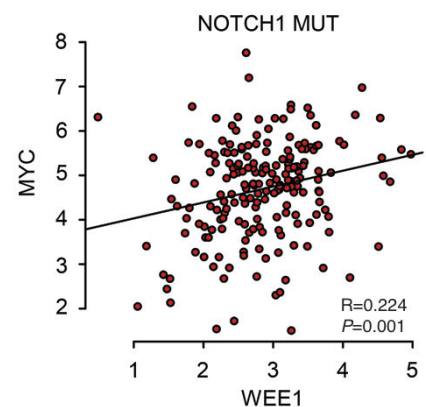

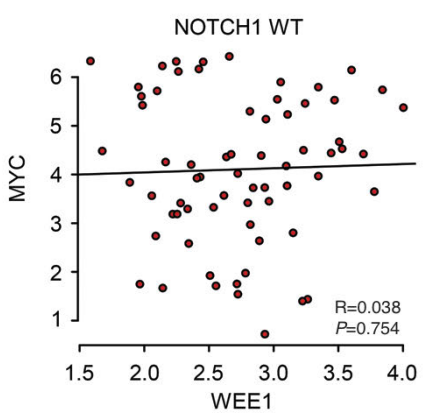

Figure 2. MYC directly activates WEE1 transcription. (A) Jurkat and CUTLL1 cells were infected with lentiviruses expressing control (shCtrl) or MYC shRNA (shMYC\#1 or shMYC\#2). WEE1 mRNA and protein levels were determined by real-time-quantitative polymerase chain reaction (RT-qPCR) and immunoblots. ACTIN served as a loading control. (B) CCRF-CEM and KOPTK1 cells were treated with JQ1 as indicated, and WEE1 protein levels were determined by immunoblots. (C) Schematic presentation of MYC binding site (E-box, -39 -34) on the WEE1 promoter. The potential MYC responsive element (wild-type, WT) and its mutant (MUT) are shown as indicated. (D) Binding of MYC to the WEE1 promoter was analyzed by chromatin immunoprecipitation (ChIP) in CUTLL1 cells. Averages of fold enrichment between MYC and isotype IgG are shown. NCL was analyzed as a positive control. (E) Luciferase reporter activities of the WEE1 promoter (-210-342bp) containing MYC RE-WT (and MUT) were detected in the presence of ectopically expressed MYC in 293T cells. 3xMYC E-box (3Ebox) sequences were used as a positive control. Reporter activities relative to empty pGL3-Basic vector (Vector) are shown. (F) Correlation of WEE1 expression with MYC in 117 primary T-cell acute lymphoblastic leukemia (T-ALL) samples (GSE26713). (G) Correlation of WEE1 expression with MYC in 264 primary T-ALL samples. (H) Correlation of WEE1 expression with MYC in NOTCH1 mutant or WT T-ALL cases shown in (G). Gene expression levels from primary T-ALL samples are presented in Log, scale. Data of RT-qPCR and luciferase reporter analysis shown represent the means ( \pm standard deviation) of biological triplicates. $* * P<0.01$. 
A

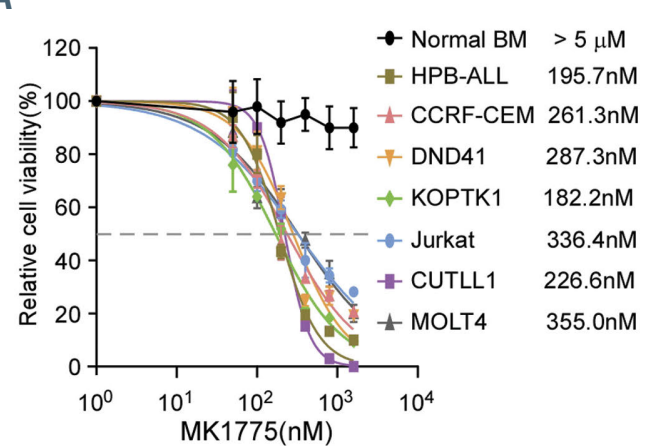

D

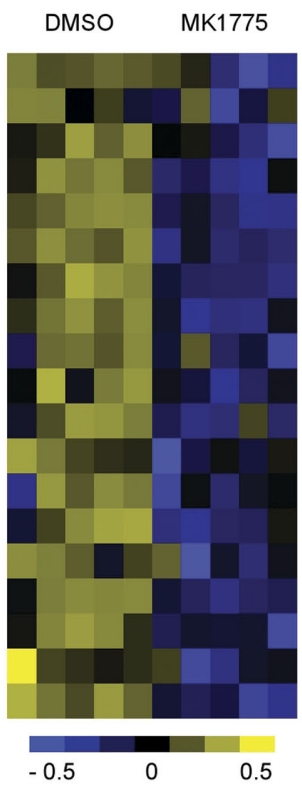

\section{3-Phosphoglycerate} Fructose-6-Phosphate Lactic acid

D-Glucosaminic acid

L-Malic acid

Fumaric acid

$\mathrm{N}$-carbarmoyl-L-Aspartate

Uridine

dTMP

Orotic acid

$\mathrm{N}$-formyl-L-Methionine

L-Aspartic acid

L-Ornithine

beta-Alanine

Glutathione

$\mathrm{N}$-acetyl-L-Leucine

(R)-3-Hydroxybutyric acid

alpha-Linolenic acid

Cholesterol

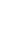

B

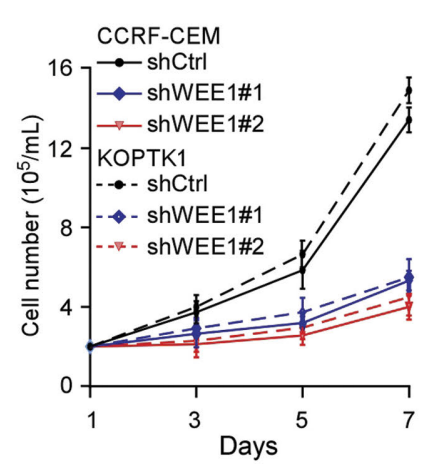

C

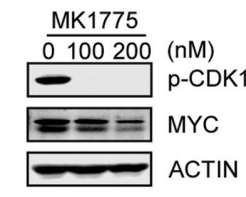

shCtrl \#1 \#2

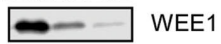

$---M Y C$

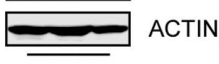

shWEE1

E

G
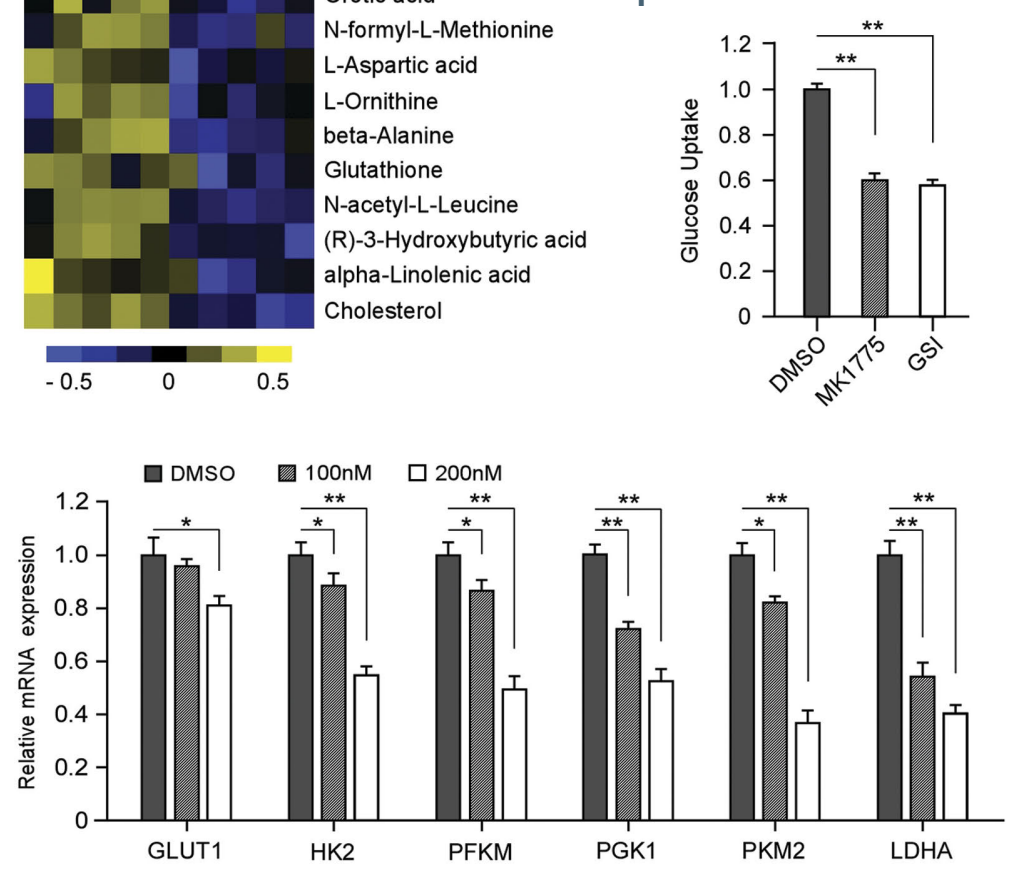

Metabolite Set Enrichment Overview

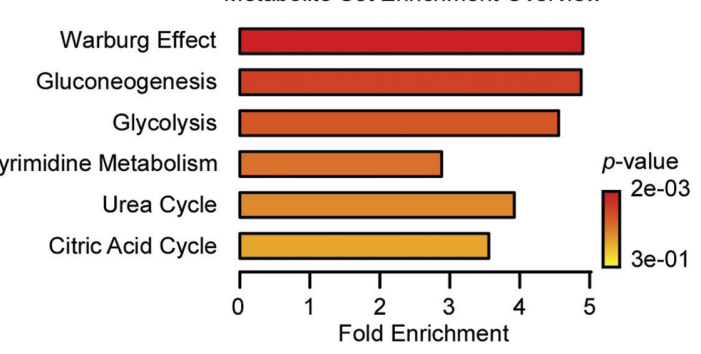

F

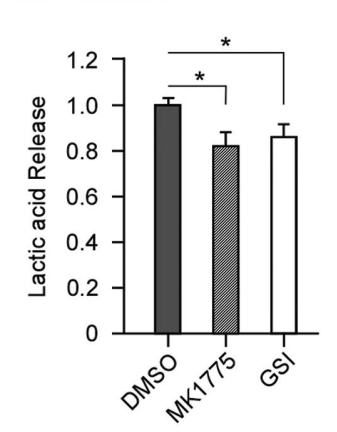

H
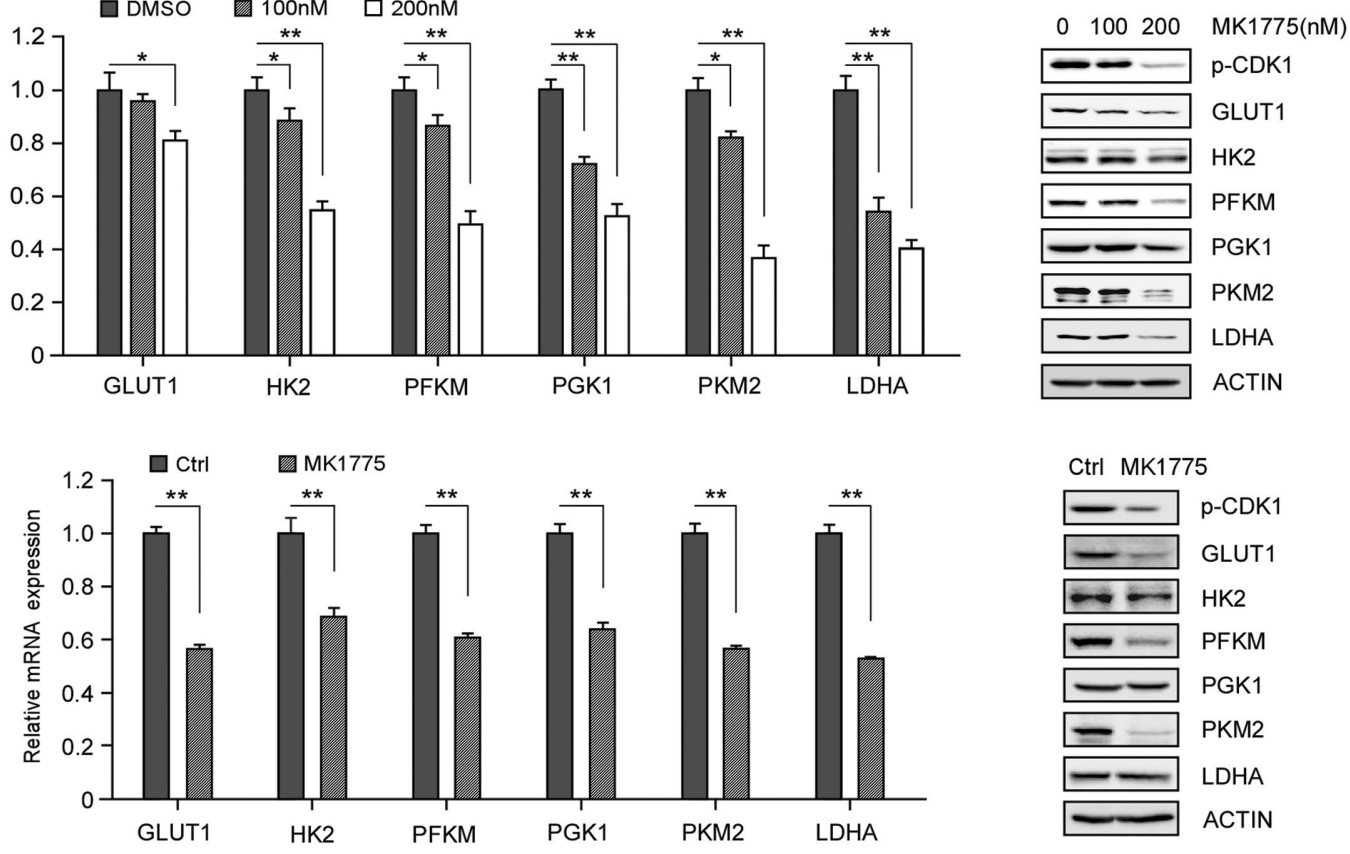

Ctrl MK1775

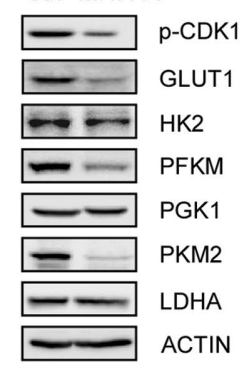


Figure 3 (from previous page). WEE1 inhibition compromises T-cell acute lymphoblastic leukemia (T-ALL) cell viability and aerobic glycolysis. (A) Cell viability analysis in multiple T-ALL cell lines subjected to MK1775 treatment at various concentrations (50, 100, 200, 400,800 and 1600 nM) for 24 hours (h). Cell viability was determined using the CCK8 Cell Proliferation Assay Kit. Percentages of viable cells are shown relative to the untreated control. (B) CCRF-CEM and KOPTK1 cells were infected with lentiviruses expressing control (shCtrl) or WEE1 (sh\#1 or sh\#2). Live cells were counted at the indicated time points and cell growth was plotted as shown. (C) HPB-ALL cells were treated with MK1775, or infected with lentiviruses expressing WEE1 shRNA (sh\#1 or sh\#2) as indicated. Phosphorylated CDK1 ( $\mathrm{p}-$ CDK1), WEE1 and MYC protein levels were analyzed by immunoblots. As a WEE1 substrate, CDK1 phosphorylation was used to reflect WEE1 activity. ACTIN serves as a loading control. (D) Heatmap of down-regulated metabolites in MK1775 (200 nM)-treated HPB-ALL cells. Intracellular metabolites were prepared and analyzed by gas chromatography time-of-flight mass spectrometer $(n=5)$. Identification of significantly different metabolites between experimental groups were determined by variable importance in projection (VIP) values (VIP $>1)$ and Student $t$-test $(P<0.05)$. Colors indicate relative metabolite abundance. $(E)$ Metabolite set enrichment analysis of significantly down-regulated metabolites $(P<0.01)$ in MK1775-treated HPB-ALL cells. (F) CUTLL1 cells were treated with MK1775 (200 nM) or GSI Compound $\mathrm{E}(1 \mu \mathrm{M})$ for $24 \mathrm{~h}$. Glucose consumption and lactate secretion were analyzed and normalized to the same live cell number. (G) HPB-ALL cells were treated with MK1775 for $24 \mathrm{~h}$ as indicated. Six representative genes involved in glycolysis pathway were analyzed by real-time quantitative polymerase chain reaction (RTqPCR) (left). (Right) Immunoblots of indicated proteins. (H) Primary cells from a T-ALL patient were injected into NPG mice which underwent control (Ctrl) or MK1775 $(20 \mathrm{mg} / \mathrm{kg})$ treatments as described in the Methods section. Human CD45+cells from the spleens of control or MK1775-treated mice were purified for RT-qPCR and immnoblotting to assess glycolytic gene expression. Data shown represent the means $( \pm$ standard deviation) of biological triplicates. $* P<0.05$, $\star \star P<0.01$. Editor's note. This figure is slightly different from the one pre-published as early view because the authors noticed an error in panel $C$ and asked to replace it with the one shown above.

but not N-MYC, activates WEE1 expression in T-ALL, and most likely in other tumor contexts as well.

Previous chromatin immunoprecipitation sequencing (ChIP)-Seq analysis manifested a strong binding signal of MYC, but not TAL1 or GATA3, in the WEE1 locus (Online Supplementary Figure S2F), ${ }^{35,36}$ further supporting the concept that MYC directly activates WEE1 transcription. To validate these data, we performed conventional ChIP assays and revealed a significant increase in MYC recruitment to the potential binding site on WEE1, similar to a well-characterized MYC target NCL (Figure 2C and D). We next constructed the WEE1 promoter containing the MYC responsive element (RE, E-box) into a luciferase reporter vector. Similar to the triple MYC RE (3 E-box) reporter as a positive control, the WT WEE1 reporter was strongly activated by MYC whereas the mutant RE with disrupted E-box was only slightly induced (Figure 2C and $\mathrm{E}$ ). The weak activation on the mutant RE was probably due to additional unrevealed MYC binding sites or other transcription factors contributing to WEE1 luciferase reporter expression. In further support of this, gene expression profiling of 117 primary $\mathrm{T}_{-} \mathrm{ALL}^{30}$ revealed a strong and significant correlation between WEE1 and MYC mRNA levels $(\mathrm{R}=0.335$, $P<0.001$ ) (Figure $2 F$ ). MYC showed a significant correlation with WEE1 ( $\mathrm{R}=0.187, P=0.002)$ in another expression profile dataset with 264 primary T-ALL (Figure $2 \mathrm{G}){ }^{32}$ Interestingly, $M Y C$ was more strongly and significantly correlated with WEE1 in NOTCH1 mutant samples $(\mathrm{R}=0.224, P=0.001)$ than NOTCH1 WT specimens $(\mathrm{R}=0.038, P=0.754$ ) (Figure $2 \mathrm{H})$. These data suggest that MYC preferentially regulates WEE1 in NOTCH1 mutant cases, and MYC probably activates differential gene expression in NOTCH1 WT and mutant T-ALL. Consistent with this, MYC depletion barely changed WEE1 expression in NOTCH1 WT SUP-T1 cells (Online Supplementary Figure S3). Our data thus provide strong evidence demonstrating that oncogenic MYC specifically binds to WEE1, particularly in NOTCH1 mutant T-ALL cells, for direct transcriptional activation.

\section{Inhibition of WEE1 impairs aerobic glycolysis}

Elevated WEE1 expression driven by MYC poses the possibility that T-ALL cells may be particularly dependent on WEE1 for cell proliferation and survival. Indeed, a selective WEE1 inhibitor MK1775 as a single agent reduced cell viability in a dose-dependent manner in seven T-ALL cell lines, whereas the effect on normal BM cells was minimal (Figure 3A). Similarly, WEE1 depletion significantly suppressed KOPTK1 and CCRF-CEM cell growth (Figure $3 \mathrm{~B}$ ). In support of our findings, MK1775 was shown to elicit ALL cell apoptosis primarily due to disruption of the G2-M cell cycle checkpoint and increased DNA damage, ${ }^{13}$ which were also detected in CUTLL1 cells (Online Supplementary Figure S4). Of particular interest, we found that WEE1 depletion or inhibition reduced MYC steady-state levels (Figure $3 \mathrm{C}$ and Online Supplementary Figure $S 5 A$ and B). Given that MYC is a master regulator in controlling cancer cell metabolism in the majority of tumor contexts, ${ }^{37}$ we reasoned that WEE1 inhibition would lead to metabolic change in T-ALL cells through MYC downregulation. To test this hypothesis, HPB-ALL cells treated with or without MK1775 were subjected to metabolomic analysis. Relative to the mock treatment, MK1775 induced global metabolic changes. Notably, WEE1 inhibition caused a decreased production of fructose-6-phosphate, 3-phosphoglycerate and lactic acid, which are crucial metabolic intermediates or products involved in aerobic glycolysis (Figure 3D). We further conducted metabolite set enrichment analysis of all down-regulated molecules and revealed that the Warburg effect was the top hit affected by WEE1 inhibition (Figure 3E). Consistent with these data, MK1775 significantly inhibited glucose uptake concomitant with a decrease in lactic acid secretion in multiple T-ALL cells, similar to GSI compound E which was previously shown to repress glycolysis in T-ALL (Figure 3F and Online Supplementary Figure S5C). ${ }^{18}$ WEE1 depletion yielded similar results to the MK1775 treatment (Online Supplementary Figure S5D). Importantly, addition of membrane soluble pyruvate, commonly encountered as one of the end products of glycolysis, significantly rescued MK1775-induced cell death (Online Supplementary Figure S5E), suggesting that the metabolic effect is one of the primary mechanisms underlying MK1775-mediated antileukemic activity.

This metabolic change is presumably due to altered MYC expression resulting from WEE1 inhibition, as enforced MYC expression significantly rescued glycolysis defects induced by MK1775 (Online Supplementary Figure S6A). Most likely, MYC acts as an important downstream player mediating the role of WEE1 in regulation of glycolysis. Consistent with previous reports that MYC activates a panel of glycolytic gene expression, ${ }^{37}$ WEE1 inhibition led to downregulation of these genes in T-ALL cells (Figure 3G and Online Supplementary Figure $S 6 B$ ). We also assessed the in vivo effect of MK1775 on glycolysis in a T-ALL patient- 
derived xenograft (PDX) derived from primary T-ALL sample \#1 (Online Supplementary Table S3). Human $\mathrm{CD} 45^{+}$leukemia cells from the spleen were analyzed for glycolysis-related gene expression. Again, MK1775 suppressed the expression of several key enzymes involved in the glycolysis pathway (Figure $3 \mathrm{H}$ ). Taken together, we identify a crucial role of WEE1 in regulation of glucose metabolism, in addition to its well-defined function in DNA damage response and cell cycle checkpoint..$^{10}$ In this regard, multiple mechanisms account for the antitumor efficacy of WEE1 inhibitors.

\section{WEE1 inactivation sensitizes T-cell acute lymphoblastic leukemia cells to glutaminase inhibitors}

Glucose and glutamine are two primary nutrients utilized by cancer cells for their proliferation and growth. We then surmised that a decrease in glycolysis may render $\mathrm{T}$ ALL cells more addicted to glutaminolysis and glutamine
A

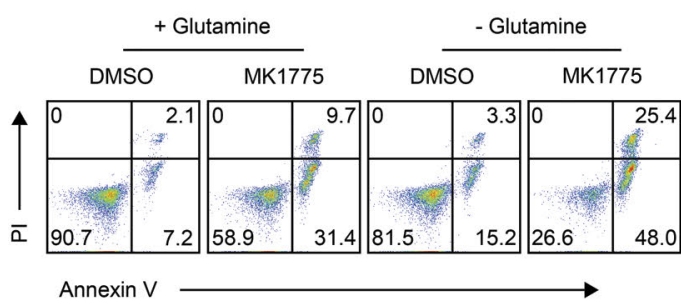

B
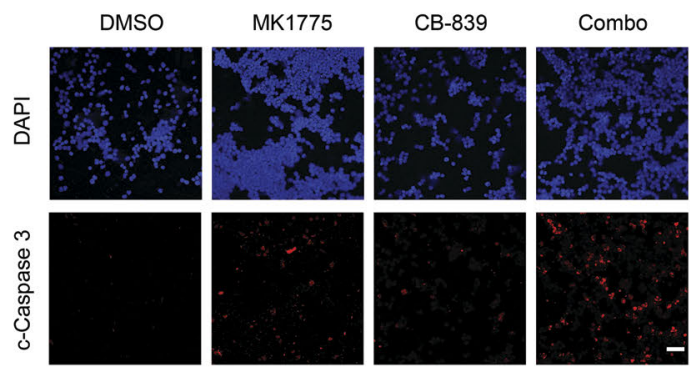
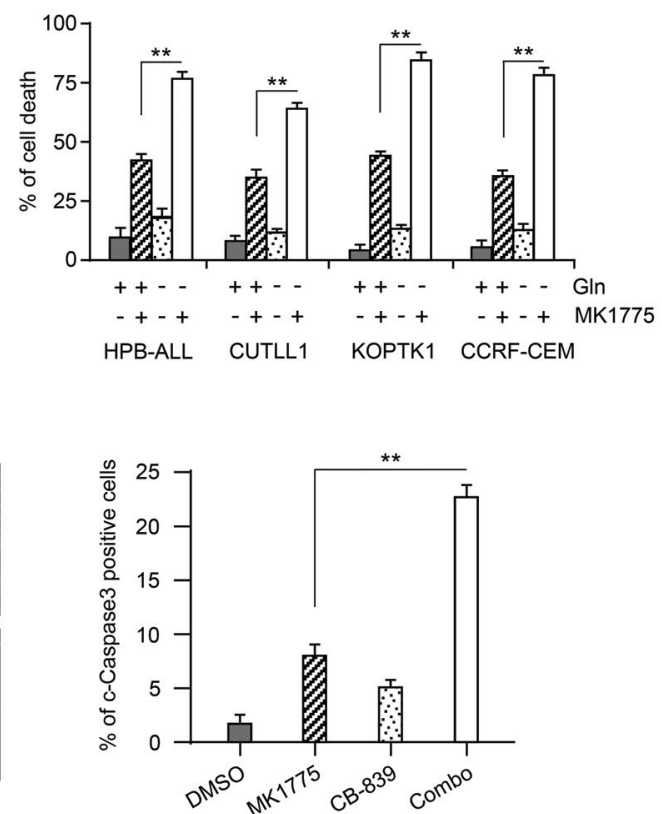

C
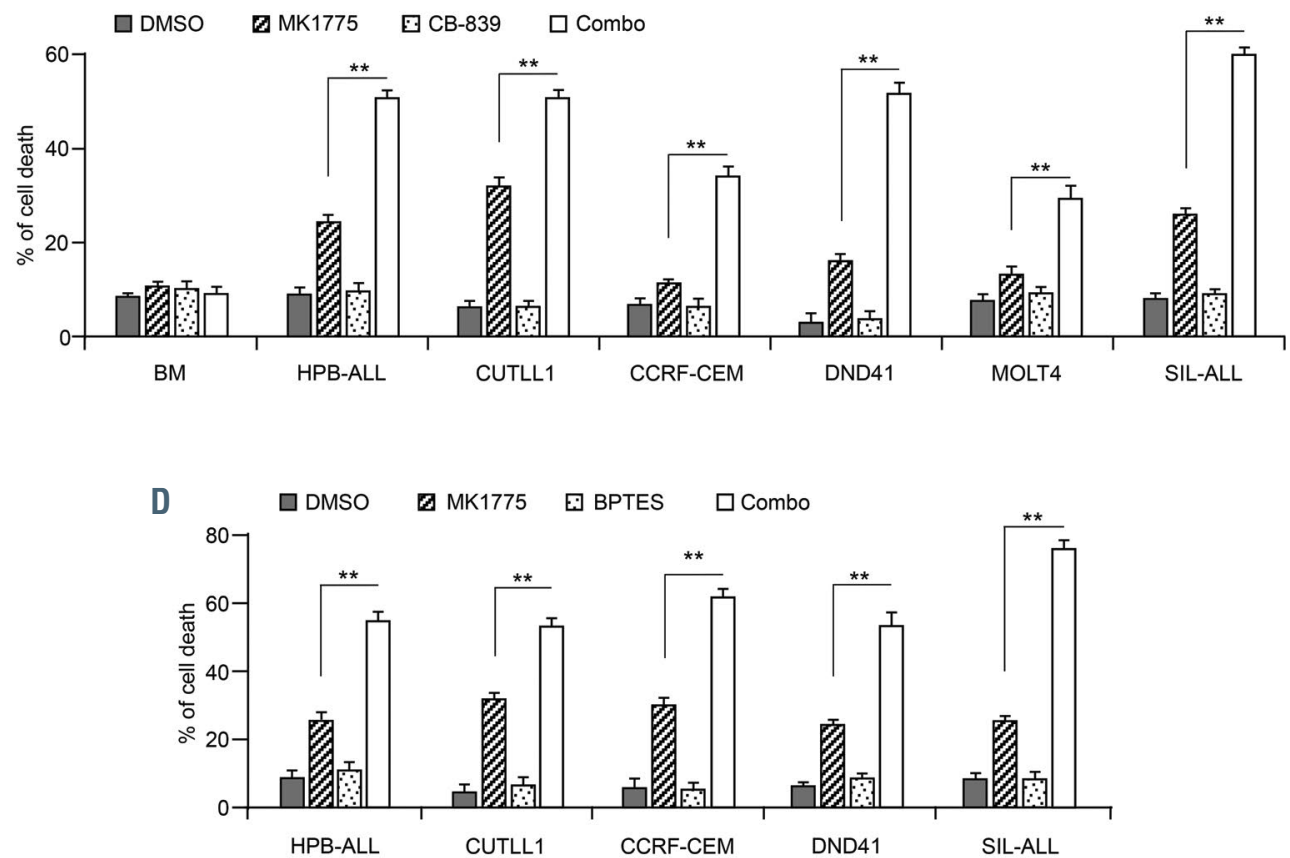

Figure 4. WEE1 inhibition sensitizes T-cell acute lymphoblastic leukemia cells to glutaminolysis inhibition. (A) T-ALL cells were treated with MK1775 (100 nM) in the presence or absence of Glutamine (Gln, $2 \mathrm{mM}$ ) for 48 hours (h). Apoptotic cell death was analyzed by Annexin V/PI staining and flow cytometry analysis. (Left) Representative flow cytometry graphs of HPB-ALL cells. (Right) Quantifications of cell death from four T-ALL lines are presented on the right. (B) Immunofluorescence images of cleaved Caspase-3 (c-caspase 3, red) and DAPI (blue) in HPB-ALL cells undergoing DMSO, MK1775(100 nM), CB-839 (100 nM) or dual treatments for 48 $\mathrm{h}$. Scale bar, $50 \mu \mathrm{m}$. (Right) Quantifications of fluorescence signals. (C) Apoptotic cell death was analyzed by Annexin V/PI staining in normal bone marrow (BM) and T-ALL cell lines after treatments as in (B). (D) Apoptotic cell death was analyzed in T-ALL cells after treatments with DMSO, MK1775 (100 nM), BPTES (10 $\mu$ M) and combination for $48 \mathrm{~h}$. Data shown represent the means ( \pm standard deviation) of biological triplicates. $* * P<0.01$. 
deficiency would synergize with WEE1 inhibition. To test this hypothesis, we treated several T-ALL cells with MK1775 in the presence or absence of glutamine in the culture medium. Glutamine deficiency induced robust cell death in combination with MK1775 treatment in multiple T-ALL cells (Figure 4A), suggesting that inhibition of glutamine uptake or metabolism exacerbates cell death induced by WEE1 inhibition. Glutaminolysis is a stepwise process by which imported glutamine is converted to glutamate (dependent on glutaminase activity) and subse- quently transformed to $\alpha$-ketoglutarate, a TCA cycle intermediate, for further catabolism and production of $\mathrm{NADH} .{ }^{20} \mathrm{GLS} 1$ is the glutaminase converting glutamine to glutamate, which is the first and rate-limiting step in the glutaminolysis pathway. We predicted that co-inhibition of WEE1 and GLS1 would disrupt the integrity of the TCA cycle, leading to metabolic catastrophe and subsequent cell death. Given that the selective GLS1 inhibitor CB-839 has been evaluated in clinical trials for anti-tumor activity, ${ }^{38,39}$ we therefore determined whether CB-839 elicited a

A

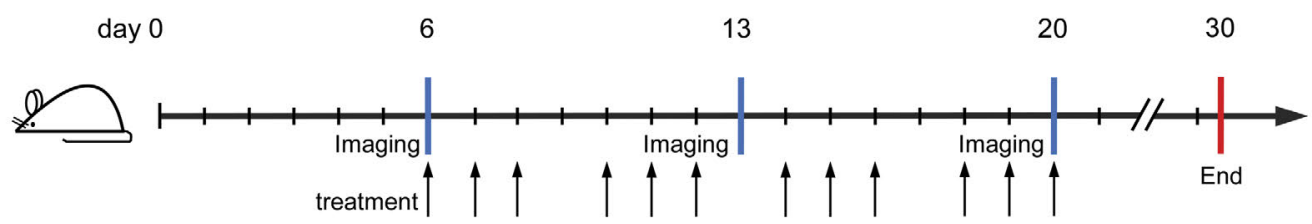

B
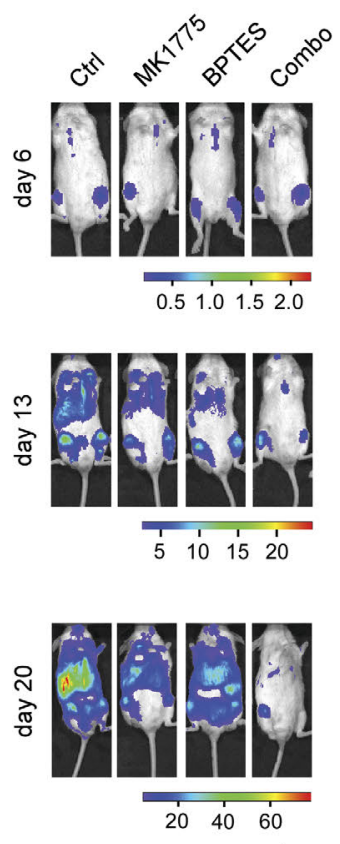

Radiance $\left(\times 10^{7}, \mathrm{p} / \mathrm{sec} / \mathrm{cm}^{2} / \mathrm{sr}\right)$
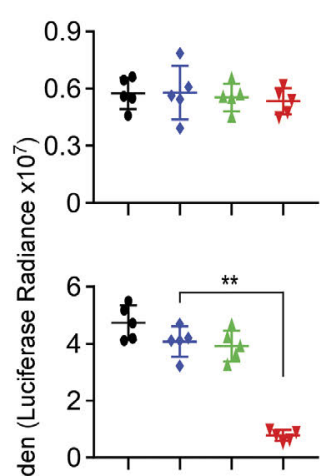

흘

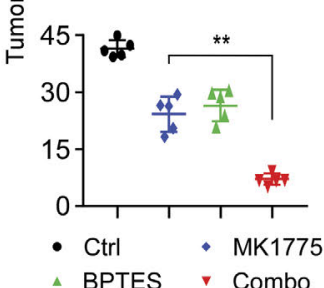

C

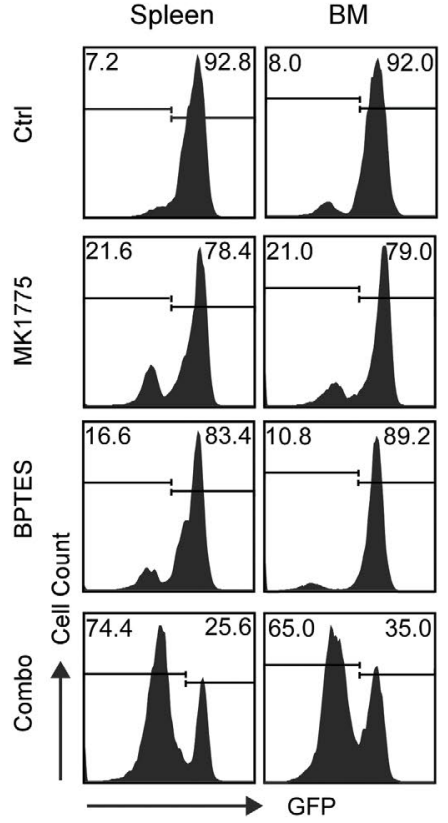

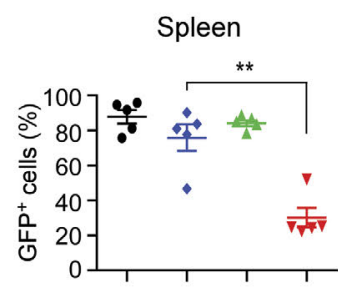

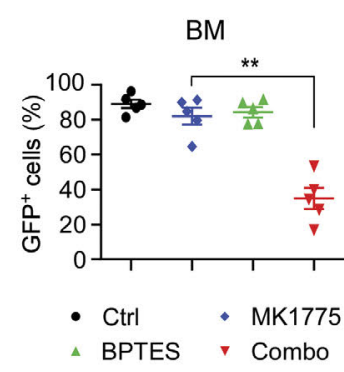

D

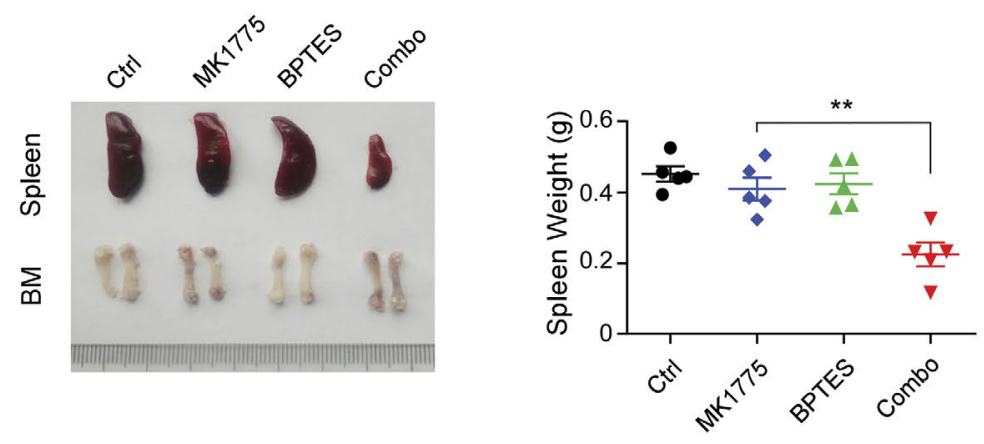

Figure 5. MK1775 and BPTES treatments demonstrate synergistic anti-leukemia efficacy in HPB-acute lymphoblastic leukemia (ALL) xenografts. (A) Graphical illustration of HPB-ALL luciferase T-cell ALL xenografts and treatment strategy (see Methods section). Single or dual treatments started at day 6 post engraftment and underwent a "three days on and one day off" schedule for four cycles as illustrated. When control mice became moribund around day 30 post engraftment, all mice were euthanized to assess leukemogenesis in vivo and therapeutic responses. (B) Representative images (left) and quantification (right) of tumor burden as assessed by luciferase luminescence signals in HPB-ALL xenografts ( $n=5$ per group). Combo: combination. (C) GFP ${ }^{+}$cells from the spleen and bone marrow were analyzed by flow cytometry (left). (Right) Data from five individual mice. (D) Representative spleen and bone images are shown (top) with spleen weights (bottom). $* * P<0.01$. 
synergistic effect with MK1775. Immunofluorescence analysis and immunoblots of cleaved Caspase-3 in HPBALL and CUTLL1 cells manifested robust apoptosis upon combined treatments as compared to single treatment (Figure 4B and Online Supplementary Figure S7). Annexin V staining confirmed strong synergism by dual treatments in six T-ALL cell lines, while sparing normal BM cells (Figure 4C). Consistently, another GLS1 inhibitor, bis-2-(5-phenylacetamido-1,3,4-thiadazol-2-yl) ethyl sulfide (BPTES), elicited synergistic cell death in combination with MK1775 (Figure 4D). Therefore, these findings indicate the strong anti-T-ALL efficacy by co-inhibition of WEE1 and GLS1.

\section{Targeting of WEE1 and glutaminase suppresses HPB-ALL xenografts}

To evaluate the in vivo efficacy of the combination strategy, we established a human xenograft using HPB-ALL cells. In order to visualize leukemia cell expansion in vivo, lentiviruses expressing firefly luciferase were infected into HPB-ALL cells and five million luciferase-expressing cells were intravenously injected into each NPG mouse. The intensities of luciferase luminescence signals were equivalent in all mice at day 6 post engraftment, and these animals were then randomly divided into four groups subjected to control, MK1775, BPTES or combination treatment (Figure 5A and B). Whereas single administration showed a moderate inhibition of leukemia progression, MK1775/BPTES combination elicited a much stronger anti-leukemia effect, as evidenced by bioimaging signals at day 13 and day 20 post xenograft (Figure 5B). When the control cohort became moribund, all the mice were sacrificed to assess the leukemia burden in vivo. Administration of either MK1775 or BPTES alone moderately inhibited engraftment, and MK1775/BPTES combination induced a synergistic and remarkable suppression of leukemogenesis (Figure 5C). As a result, dual treatment ameliorated spleen enlargement and resulted in more reddish bones (Figure

A
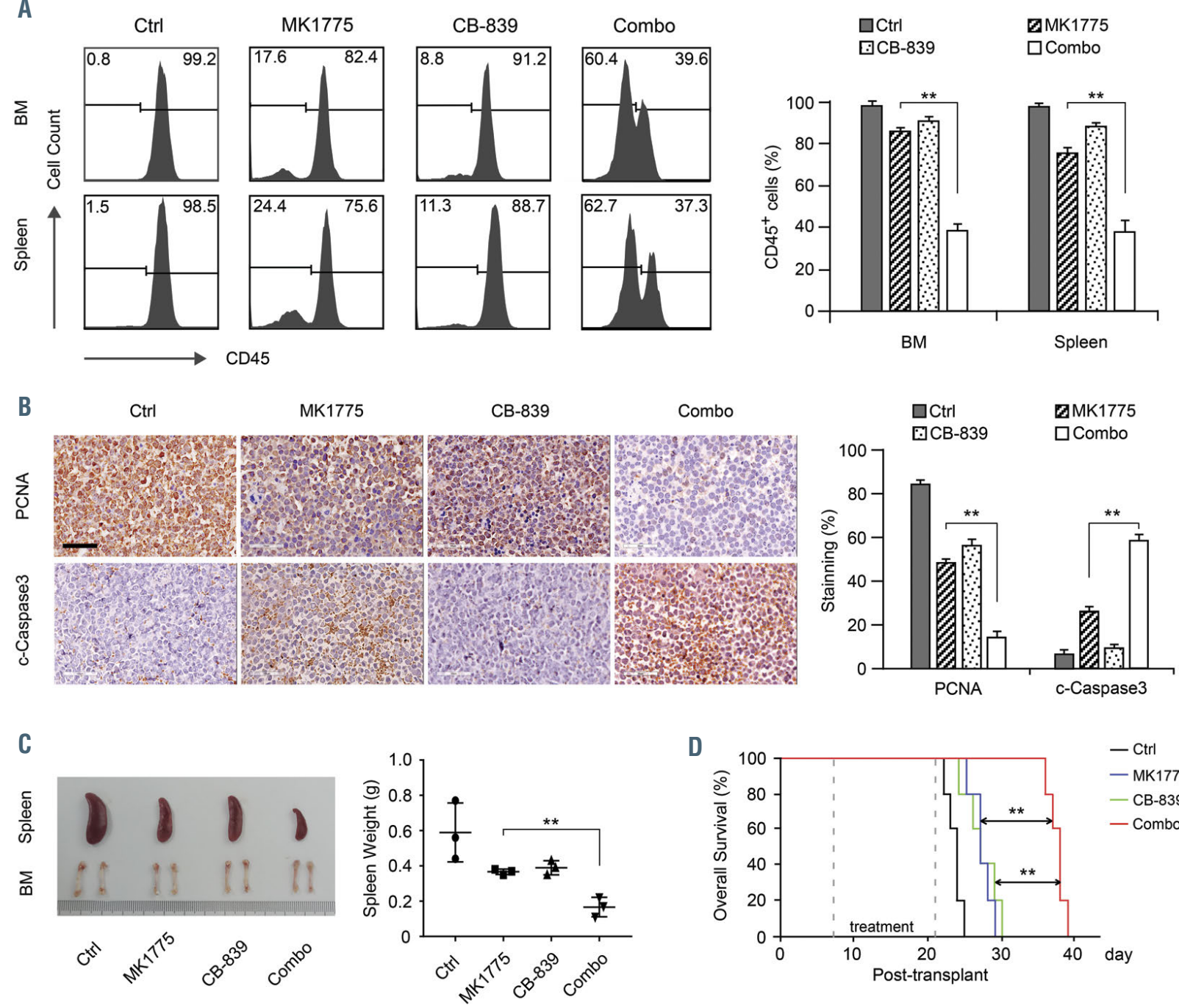

D

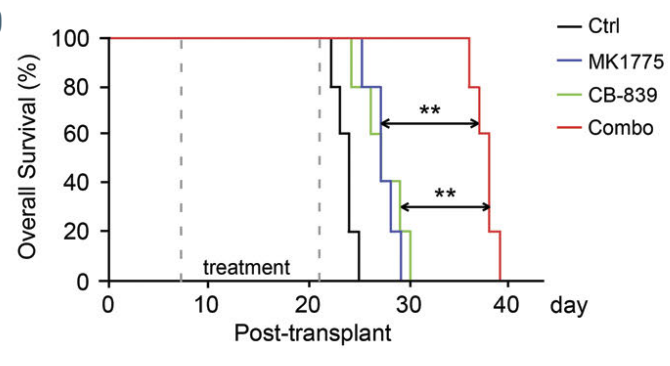

Figure 6. Synergistic anti-leukemia effects of MK1775 and CB-839 on a patient-derived T-cell acute lymphoblastic leukemia (T-ALL) xenograft. Human primary T-ALL cells were injected into irradiated NPG mice. (A) Human CD45 cells from bone marrow (BM) and spleen were analyzed by flow cytometry when the controltreated mice became moribund around day 25 post xenograft. (Right) Quantifications of CD45+ percentages. Combo: combination. (B) Representative immunohistological images of PCNA and cleaved Caspase-3 (c-Caspase 3) in the spleen sections from mice receiving different treatments. Scale bar, $50 \mu \mathrm{m}$. (A and B) Quantifications shown represent the means ( \pm standard deviation) of biological triplicates. (C) Representative spleen and bone images of mice subjected to different treatments (left) with spleen weights (right). (D) Kaplan-Meier survival curves of T-ALL PDX treated with MK1775 and/or CB-839 ( $n=5$ in each group). Dotted lines define the treatment time frame. $* * P<0.01$. 
5D). These findings corroborate the anti-T-ALL efficacy of MK1775/BPTES combination in vivo.

We also evaluated the toxicity of the drug combination in healthy C57/BL6 mice using the same treatment strategy as shown in Figure 5A. Body weights of these mice were comparable to the control cohort (Online Supplementary Figure S8A). Analysis of blood parameters at the end of the treatment revealed a mild decrease in red and white cell counts (Online Supplementary Figure S8B), and the decline in blood cell counts vanished two weeks after treatment ended (Online Supplementary Figure S8C). Moreover, hematoxylin \& eosin (H\&E) staining revealed undetectable damage in various organs (Online Supplementary Figure S8D). Consistently, healthy human BM cells were not sensitive to dual treatment either (Figure 4C), arguing of manageable toxicity of this combination strategy. This is probably due to lower WEE1 and MYC expression in the majority of normal cells. Therefore, therapeutic targeting of WEE1 may not induce significant inhibition of MYC and glycolysis as much as it does in tumor cells.

\section{Combined WEE1/GLS1 inhibition prolongs overall survival in a T-cell acute lymphoblastic leukemia patient-derived xenograft}

Given CB-839 is currently being evaluated in clinical trials ${ }^{39}$ we next explored the translational potential of MK1775 in combination with CB-839 in a T-ALL PDX. Human $\mathrm{CD} 45^{+}$leukemia cell distributions in vivo were analyzed to assess tumor burden and therapeutic responses. Again, the combination treatment induced synergistic tumor growth inhibition compared with the control or monotherapy, as evidenced by much lower human CD45+ tumor cell percentages (Figure 6A). Immunohistological (IHC) analysis of the spleen sections further confirmed reduced cell proliferation and massive intratumoral apoptosis due to administration of both compounds, as quantified by attenuated proliferating cell nuclear antigen (PCNA) and increased cleaved Caspase-3 staining, respectively (Figure 6B). Injection of MK1775 and CB-839 resulted in much smaller spleen size and more reddish bones (Figure 6C). More importantly, dual treatments significantly prolonged the lifespans of the T-ALL PDX as compared to single treatment (Figure 6D). Together with the preclinical studies in HPB-ALL xenografts, these results strongly suggest the clinical potential of WEE1/GLS1 inhibitors as a promising T-ALL targeted therapeutics.

\section{Discussion}

Intensified T-ALL chemotherapies face the challenges of significant side effects, frequent relapses, and drug resistance. To improve the treatment of T-ALL and reduce associated toxicity, introduction of new targeted agents is desperately needed. We here show that WEE1 kinase is a promising therapeutic target. Reduced cell viability upon WEE1 inhibitor MK1775 treatment is partly attributable to significant suppression of aerobic glycolysis, leading to TALL cells more addicted to glutaminolysis. Administration of WEE1 and GLS1 inhibitors induces synergistic lethality in T-ALL cells and leukemia xenografts. These results highlight a promising therapeutic strategy of dual targeting of cell cycle kinase and metabolic enzymes in T-ALL treatments.
Loss of cell cycle control plays a prominent role in the pathogenesis of T-ALL. The tumor suppressors $p 16^{\text {INK } 4 A}$ and p14 $4^{\mathrm{ARF}}$ encoded by the CDKN2A locus are frequently lost in T-ALL due to chromosomal deletions. ${ }^{40} \mathrm{p} 16^{\mathrm{INKA}}$ and p14 ${ }^{\mathrm{ARF}}$ inactivates the cyclin D1-CDK4/6 and cyclin ECDK2 complexes, respectively, leading to G1-S arrest for DNA repair. ${ }^{9}$ As such, loss of CDKN2A results in overactivation of these CDK complexes, enabling T-ALL cells to enter $\mathrm{S}$ phase for replication despite DNA damage. T-ALL cells, with a deficient G1-S checkpoint, are therefore more reliant on the G2-M cell cycle checkpoint to prevent excessive DNA damage that may lead to mitotic catastrophe and cell death. In support of this notion, we and others have shown that WEE1 expression is significantly increased in a variety of T-ALL cell lines and patientderived primary cells. ${ }^{13} \mathrm{We}$ further delineate the molecular mechanism underlying WEE1 expression in T-ALL by identifying MYC as a prominent regulator directly activating the WEE1 transcription. MYC-mediated WEE1 upregulation is also found in Burkitt's lymphoma cells, suggesting the MYC-WEE1 axis as a general regulatory mode governing WEE1 expression in human cancers.

Inhibition of WEE1 in cancer cells circumvents cell cycle arrest during the G2 phase and enables cell division despite accumulation of DNA damage. T-ALL cells crucially depend on the G2 checkpoint in the presence of DNAdamage inducing drugs. WEE1 inhibitor manifested synergistic anti-leukemic activity in combination with cytarabine or olaparib. ${ }^{13,41,42}$ We here identify an additional metabolic vulnerability of T-ALL cells in response to WEE1 inhibition after which they become particularly addicted to glutaminolysis for cell survival. The underlying mechanism involves MK1775-mediated glycolytic suppression at least in part via downregulation of MYC, a master regulator in controlling glucose metabolism in the majority of tumor contexts. ${ }^{37}$ Indeed, we demonstrate that overexpression of MYC significantly rescued glycolysis defect due to MK1775 treatment, suggesting that MYC acts as an important downstream player mediating the role of WEE1 in the regulation of glycolysis. It is interesting to note that although a previous report suggests that MYC promotes glutaminolysis as well by activating GLS1 expression in B lymphoma and prostate cancer cells, ${ }^{43}$ neither MYC downregulation nor WEE1 inhibition affected GLS1 expression in the context of T-ALL (data not shown). Moreover, WEE1 inhibition switches T-ALL cells to a more glutamine-dependent state such that simultaneous suppression of glycolysis and glutaminolysis by MK1775 and GLS1 inhibitor respectively induced potent synergistic anti-T-ALL effects (Online Supplementary Figure S9).

Our findings reveal a molecular link between cell cycle and cancer metabolism by demonstrating the contribution of WEE1 to glycolysis. WEE1 inhibition, which unleashes the G2-M checkpoint and accelerates cell cycle, reprograms the cellular metabolism such that tumor cells with decreased glycolysis become more addicted to glutaminolysis. These results are reminiscent of a recent finding that activation of cyclin D1-CDK4/6 by dysregulation of Fbxo4-cyclin D1 axis leads to cellular dependency on glutamine metabolism and sensitizes tumor cells to CB-839.44 As such, increased glutamine dependency could be a consequence of over-activated CDK complex, which provides a potential therapeutic opportunity in fast-dividing tumor cells. Loss of cell cycle checkpoints due to genetic mutations and/or utilizing checkpoint inhibitors in cancer treat- 
ment may sensitize tumor cells to GLS1 inhibitors. Taken together, our findings support further investigation of MK1775 and CB-839 combination in clinical settings for T-ALL treatments, given that the respective monotherapy has been evaluated in multiple clinical trials and shows tolerable toxicity. $39,45,46$

\section{Disclosures}

No conflicts of interest to disclose.

\section{Contributions}

HL conceived and designed the study; HL supervised the study. $H L, J H$ and $T W$ wrote the manuscript; JH and TW performed the majority of experiments; $J X, L W, H S, J J, M Y, J W$ and $D W$ provided technical support; SW, $P L$ and FZ provided primary T-ALL samples; YL helped with data analysis of primary T-ALL samples; $H L$ and $G Q$ analyzed and interpreted the data.

\section{Acknowledgments}

The authors would like to thank Liu Lab members for technical support and critical reading of the manuscript, the Core Facility of Medical Research Institute at Wuhan University for immunofluorescence, flow cytometry and histological analysis.

\section{Funding}

This research was supported by grants from National Key R\&D Program of China (2017YFA0505600 to GQ), National Natural Science Foundation of China (81770177, 81970152 to HL, 81803003 to MY), National Science Fund for Distinguished Young Scholars (81725013 to GQ), Hubei Provincial Natural Science Fund for Distinguished Young Scholars (2017CFA072 to HL), Hubei Provincial Natural Science Fund for Creative Research Groups (2018CFA018 to GQ), and Innovative Research Grants from Wuhan University (2042019kfo338 to HL and $2042017 \mathrm{kfO} 282$ to GQ).

\section{References}

1. Belver L, Ferrando A. The genetics and mechanisms of $\mathrm{T}$ cell acute lymphoblastic leukaemia. Nat Rev Cancer. 2016;16(8):494507.

2. Van Vlierberghe P, Ferrando A. The molecular basis of $\mathrm{T}$ cell acute lymphoblastic leukemia. J Clin Invest. 2012;122(10):33983406.

3. Hunger SP, Lu X, Devidas M, et al. Improved survival for children and adolescents with acute lymphoblastic leukemia between 1990 and 2005: a report from the Children's Oncology Group. J Clin Oncol. 2012; 30(14):1663-1669.

4. Inaba H, Greaves M, Mullighan CG. Acute lymphoblastic leukaemia. Lancet. 2013; 381(9881):1943-1955.

5. Bhojwani D, Pui C-H. Relapsed childhood acute lymphoblastic leukaemia. Lancet Oncol. 2013;14(6):e205-e217.

6. Litzow MR, Ferrando AA. How I treat T-cell acute lymphoblastic leukemia in adults. Blood. 2015;126(7):833-841.

7. Palomero T, Ferrando A. Therapeutic targeting of NOTCH1 signaling in T-cell acute lymphoblastic leukemia. Clin Lymphoma Myeloma. 2009;9(Suppl 3):S205-S210.

8. Chen T, Stephens PA, Middleton FK, Curtin NJ. Targeting the S and G2 checkpoint to treat cancer. Drug Discov Today. 2012;17(56):194-202.

9. Otto T, Sicinski P. Cell cycle proteins as promising targets in cancer therapy. Nat Rev Cancer. 2017;17(2):93-115.

10. Matheson CJ, Backos DS, Reigan P. Targeting WEE1 kinase in cancer. Trends Pharmacol Sci. 2016;37(10):872-881.

11. Magnussen GI, Holm R, Emilsen E, Rosnes AK, Slipicevic A, Florenes VA. High expression of Wee1 is associated with poor disease-free survival in malignant melanoma: potential for targeted therapy. PLoS One. 2012;7(6):e38254.

12. Mir SE, De Witt Hamer PC, Krawczyk PM, et al. In silico analysis of kinase expression identifies WEE1 as a gatekeeper against mitotic catastrophe in glioblastoma. Cancer Cell. 2010;18(3):244-257.

13. Ghelli Luserna Di Rora A, Beeharry N, Imbrogno $\mathrm{E}$, et al. Targeting WEE1 to enhance conventional therapies for acute lymphoblastic leukemia. J Hematol Oncol. 2018;11(1):99.

14. Slipicevic A, Holth A, Hellesylt E, Trope CG,
Davidson B, Florenes VA. Wee1 is a novel independent prognostic marker of poor survival in post-chemotherapy ovarian carcinoma effusions. Gynecol Oncol. 2014; 135(1):118-124.

15. Magnussen GI, Hellesylt E, Nesland JM, Trope CG, Florenes VA, Holm R. High expression of wee1 is associated with malignancy in vulvar squamous cell carcinoma patients. BMC Cancer. 2013;13:288.

16. Pavlova NN, Thompson CB. The emerging hallmarks of cancer metabolism. Cell Metab. 2016;23(1):27-47.

17. Zhang J, Pavlova NN, Thompson CB. Cancer cell metabolism: the essential role of the nonessential amino acid, glutamine. EMBO J. 2017;36(10):1302-1315.

18. Palomero T, Sulis ML, Cortina $M$, et al. Mutational loss of PTEN induces resistance to NOTCH1 inhibition in T-cell leukemia. Nat Med. 2007;13(10):1203-1210.

19. Herranz D, Ambesi-Impiombato A, Sudderth J, et al. Metabolic reprogramming induces resistance to anti-NOTCH1 therapies in $\mathrm{T}$ cell acute lymphoblastic leukemia. Nat Med. 2015;21(10):1182-1189.

20. Hensley CT, Wasti AT, DeBerardinis RJ. Glutamine and cancer: cell biology, physiology, and clinical opportunities. J Clin Invest. 2013;123(9):3678-3684

21. Wang Z, Hu Y, Xiao D, et al. Stabilization of Notch1 by the Hsp90 chaperone is crucial for T-cell leukemogenesis. Clin Cancer Res. 2017;23(14):3834-3846.

22. Su H, Hu J, Huang L, et al. SHQ1 regulation of RNA splicing is required for T-lymphoblastic leukemia cell survival. Nat Commun. 2018;9(1):4281.

23. Hirai H, Iwasawa Y, Okada M, et al. Smallmolecule inhibition of Wee1 kinase by MK1775 selectively sensitizes p53-deficient tumor cells to DNA-damaging agents. Mol Cancer Ther. 2009;8(11):2992-3000.

24. Yang C, Hao R, Du X, Wang Q, Deng Y, Sun $R$. Response to different dietary carbohydrate and protein levels of pearl oysters (Pinctada fucata martensii) as revealed by GC-TOF/MS-based metabolomics. Sci Total Environ. 2019;650(Pt 2):2614-2623.

25. Yue M, Jiang J, Gao P, Liu H, Qing G. Oncogenic MYC activates a feedforward regulatory loop promoting essential amino acid metabolism and tumorigenesis. Cell Rep. 2017;21(13):3819-3832.

26. Kind T, Wohlgemuth G, Lee DY, et al. FiehnLib: mass spectral and retention index libraries for metabolomics based on quadru- ple and time-of-flight gas chromatography/mass spectrometry. Anal Chem. 2009; 81(24):10038-10048.

27. $\mathrm{Hu}$ Y, Su H, Liu C, et al. DEPTOR is a direct NOTCH1 target that promotes cell proliferation and survival in T-cell leukemia. Oncogene. 2017;36(8):1038-1047.

28. Andersson A, Ritz C, Lindgren D, et al. Microarray-based classification of a consecutive series of 121 childhood acute leukemias: prediction of leukemic and genetic subtype as well as of minimal residual disease status. Leukemia. 2007; 21(6):1198-1203.

29. Coustan-Smith E, Song G, Clark C, et al. New markers for minimal residual disease detection in acute lymphoblastic leukemia. Blood. 2011:117(23):6267-6276.

30. Homminga I, Pieters R, Langerak AW, et al. Integrated transcript and genome analyses reveal NKX2-1 and MEF2C as potential oncogenes in $\mathrm{T}$ cell acute lymphoblastic leukemia. Cancer Cell. 2011;19(4):484-497.

31. Ghandi M, Huang FW, Jane-Valbuena J, et al. Next-generation characterization of the Cancer Cell Line Encyclopedia. Nature. 2019;569(7757):503-508.

32. Liu Y, Easton J, Shao Y, et al. The genomic landscape of pediatric and young adult T-lineage acute lymphoblastic leukemia. Nat Genet. 2017;49(8):1211-1218.

33. Delmore JE, Issa GC, Lemieux ME, et al. BET bromodomain inhibition as a therapeutic strategy to target c-Myc. Cell. 2011; 146(6):904-917

34. Puissant A, Frumm SM, Alexe G, et al Targeting MYCN in neuroblastoma by BET bromodomain inhibition. Cancer Discov. 2013;3(3):308-323.

35. Kourtis N, Lazaris C, Hockemeyer K, et al. Oncogenic hijacking of the stress response machinery in $\mathrm{T}$ cell acute lymphoblastic leukemia. Nat Med. 2018;24(8):1157-1166.

36. Sanda T, Lawton LN, Barrasa MI, et al. Core transcriptional regulatory circuit controlled by the TAL1 complex in human T cell acute lymphoblastic leukemia. Cancer Cell. 2012; 22(2):209-221.

37. Hsieh AL, Walton ZE, Altman BJ, Stine ZE, Dang CV. MYC and metabolism on the path to cancer. Semin Cell Dev Biol. 2015;43:11 21.

38. Song M, Kim SH, Im CY, Hwang HJ. Recent development of small molecule glutaminase inhibitors. Curr Top Med Chem. 2018;18(6): 432-443.

39. DeMichele A, Harding JJ, Telli ML, et al. 
Phase 1 study of CB-839, a small molecule inhibitor of glutaminase (GLS) in combination with paclitaxel $(\mathrm{Pac})$ in patients (pts) with triple negative breast cancer (TNBC). J Clin Oncol. 2016;34(Suppl 15):1011-1011.

40. Girardi T, Vicente C, Cools J, De Keersmaecker $\mathrm{K}$. The genetics and molecular biology of T-ALL. Blood. 2017;129(9): 1113-1123.

41. Ford JB, Baturin D, Burleson TM, Van Linden AA, Kim YM, Porter CC. AZD1775 sensitizes $\mathrm{T}$ cell acute lymphoblastic leukemia cells to cytarabine by promoting apoptosis over DNA repair. Oncotarget. 2015;6(29):28001-28010.
42. Garcia TB, Snedeker JC, Baturin D, et al. A small-molecule inhibitor of WEE1, AZD1775, synergizes with olaparib by impairing homologous recombination and enhancing DNA damage and apoptosis in acute leukemia. Mol Cancer Ther. 2017; 16(10):2058-2068.

43. Gao P, Tchernyshyov I, Chang TC, et al. cMyc suppression of $\mathrm{miR}-23 \mathrm{a} / \mathrm{b}$ enhances mitochondrial glutaminase expression and glutamine metabolism. Nature. 2009; 458(7239):762-765

44. Qie S, Yoshida A, Parnham S, et al. Targeting glutamine-addiction and overcoming CDK 4/6 inhibitor resistance in human esophageal squamous cell carcinoma. Nat Commun. 2019;10(1):1296.

45. Leijen S, van Geel RM, Pavlick AC, et al. Phase I study evaluating WEE1 inhibitor AZD1775 as monotherapy and in combination with gemcitabine, cisplatin, or carboplatin in patients with advanced solid tumors. J Clin Oncol. 2016;34(36):4371 4380.

46. Do K, Wilsker D, Ji J, et al. Phase I study of single-agent AZD1775 (MK-1775), a Wee1 kinase inhibitor, in patients with refractory solid tumors. J Clin Oncol. 2015;33(30): 3409-3415. 